\title{
SUBSÍDIOS PARA O ESTUDO DO IMPACTO DO ENSINO SUPERIOR NO PENSAMENTO DOS ALUNOS: UM ESTUDO REALIZADO NA UNIVERSIDADE DE COIMBRA
}

\author{
Emilia da Conceição Figueiredo Martins ${ }^{1}$
}

Resumo: O interesse científico pelo impacto do ensino superior no desenvolvimento dos estudantes afirma-se, progressivamente, quer pela acessibilidade da população alvo, quer pela importância atribuída àquele contexto no desenvolvimento do jovem adulto/adulto emergente. $\mathrm{O}$ estudo transversal aqui apresentado sobre o impacto da frequência do ensino superior no pensamento pós-formal, em alunos da Universidade de Coimbra (caloiros e finalistas), põe em evidência um impacto positivo no constructo de desenvolvimento intelectual e ético definido por Perry (1970), que se traduz na superação do dualismo e em melhorias significativas no relativismo. O género apresenta-se como uma variável que permite distinguir as performances cognitivas dos alunos, favorecendo as raparigas, e os resultados também são distintos nos diferentes estabelecimentos de ensino considerados.

Palavras-chave: ensino superior, desenvolvimento cognitivo, pensamento pós-formal, jovem adulto

Contribution to the study of the cognitive impact of higher education on students: A study held at the University of Coimbra (Abstract): The scientific interest on the impact of Higher Education on students has undergone a growing development not only because of the availability of its target group, but also through the importance given to that context on the development of the youngster/adult. This horizontal study about the impact of Higher Education on the post-formal reasoning in students of the University of Coimbra (freshmen and finalists) highlights a positive impact on the construct of intellectual and ethical development defined by Perry (1970), that is evident in the overcoming of dualism and a better performance as far as relativism is concerned. Gender appears as a variable that allows making a distinction in students' cognitive performances, favouring female students, and the results are also distinct in the different institutions analysed.

Keywords: higher education, cognitive development, post-formal reasoning, youth

\footnotetext{
${ }^{1}$ Escola Superior de Educação do Instituto Politécnico de Viseu - área científica de Psicologia

e-mail: emiliamartins@esev.ipv.pt
} 


\section{Introdução}

O crescente interesse da literatura, desde meados do século passado, pela faixa de desenvolvimento humano que incide sobre os estudantes do ensino superior, em muito se deve a uma confluência de factores e circunstâncias. Por um lado, existe o contributo decisivo da perspectiva do ciclo de vida (life-span) no desenvolvimento humano, que chama a atenção para o período além da adolescência; nesse sentido, são vários os autores que classificam de forma diferenciada as etapas da vida humana, consoante a perspectiva em que se colocam (Erikson, 1959, 1968; Havighurst 1973; Köhlberg 1971, 1979). Muitos outros autores (Gould, 1978; Levinson, 1978; Loevinger, 1976) contribuíram, decisivamente, para a valorização do estudo jovem adulto, como etapa onde ocorrem mudanças significativas, ao nível das interacções sociais, sexuais, vocacionais e profissionais (Pinheiro, 2003). Por outro lado, ainda que o jovem adulto não se esgote no estudante do ensino superior, esta população encontra-se mais acessível à investigação, uma vez que os estabelecimentos de ensino superior têm a dupla vantagem de concentrar os investigadores e a população em estudo. Não podemos, contudo, tomar a parte pelo todo e, nesse sentido, é preferível falar do desenvolvimento do estudante do ensino superior do que falar do desenvolvimento do jovem adulto.

Com efeito, conseguimos identificar as tarefas e os desafios singulares que se exigem a quem prossegue estudos, desde a tomada de decisão a todo o processo de selecção e colocação no ensino superior, passando pelo afastamento da família, na maioria dos casos e pela adaptação a uma nova localidade, a novos colegas, a um novo tecido social e institucional, a novos métodos de ensino e estendendo-se à experiência de interacções de carácter e natureza diferentes. Segundo Arnett $(2000,2002,2004)$, o período do adulto emergente (entre os 18 e os 25 anos) caracteriza-se por mudanças importantes na vida do adolescente anterior, onde a saída de casa sem assumir papéis sociais pré-definidos possibilita uma condição de liberdade e oportunidade para experimentar vários caminhos no amor, na educação e no trabalho, mas também de instabilidade. Na caminhada para o adulto pleno (full adulthood), o jovem estudante aprende a ser auto-suficiente e assume responsabilidades e papéis sociais característicos do adulto. As características particulares do contexto podem privilegiar a forma como é resolvida a transição e o êxito na mesma. No entanto, a literatura alerta para o reverso da medalha e a possibilidade de o contexto se constituir como fonte de pressão, potencialmente stressante, em que se pode dar uma viragem ou consolidação dos níveis de desenvolvimento atingidos até ao momento (Almeida \& Santos, 1999: Hood \& Ferreira, 1983; Martins, 2007; Pinheiro, 2003). 
Keniston $(1965,1971)$ foi dos primeiros autores americanos a preocupar-se com o desenvolvimento neste contexto, realçando o efeito mediador e moderador da origem da família (family background), relativamente à sub-cultura do ensino secundário, ao mesmo tempo que se perde essa influência familiar pela redução do contacto e aumentam outras influências sociais. Os novos desafios e tarefas do jovem adulto podem levá-lo, tanto a assumir compromissos ponderados e inseridos no processo de aculturação, como ao afastamento, indiferença e recusa de socialização. Não obstante, já entre as décadas de 30 e 50, nos Estados Unidos, se realizaram alguns trabalhos sobre o impacto do ensino superior nos valores morais dos estudantes (Learned \& Wood, 1938; NewCombs, 1943; Jacob, 1957; cit. por Pascarella \& Terenzini, 1991). Entretanto, os estudos que incidem na população de alunos que frequenta o ensino superior têm-se multiplicado. No domínio psicossocial, realçam-se os que se basearam nos trabalhos de Erikson, tais como Sanford (1962; 1966), Heath (1965), Márcia (1966), Chickering (1969), Josselson (1987) e Chickering e Reisser (1993). É mesmo aos trabalhos de Sanford $(1962 ; 1966 ; 1967)$ e da sua equipa da Universidade de Vassar que se tem atribuído o berço da pesquisa neste campo. Do ponto de vista cognitivo, destacamos Baxter-Magolda (1992), Belenky, Clinchy, Goldberger e Tarule (1986), Kitchener e King (1981), Kohlberg (1979), Loevinger (1976) e Perry (1970, 1981). A partir da década de 80 assiste-se, definitivamente, ao salto quantitativo e qualitativo, favorecido pelo desenvolvimento nas técnicas e instrumentos de recolha e tratamento de dados e pelos avanços nos desenhos de investigação.

A temática adquire maior relevo se pensarmos na mudança de paradigma subjacente ao ensino superior e suas funções que vem a ocorrer nos últimos 10 anos no mundo industrializado. Com efeito, mais do que formar perfis profissionais com vista ao desempenho de uma actividade específica, importa formar indivíduos com competências transferíveis, de natureza social, emocional, ética e cognitiva (Rovira, 2001), que lhes permitam fazer face a um mundo em transformação alucinante, sustentado na Aldeia Global para que caminhamos. $\mathrm{O}$ ensino superior não pode, pois, alhear-se desta realidade e a prova disso é o esforço traduzido no compromisso europeu de Bolonha. Já Knefelkamp, Widick e Parker (1978) realçaram a necessidade de perspectivar a finalidade e objectivos do ensino superior numa dimensão que valoriza o aluno de forma holística, enquanto indivíduo em desenvolvimento. Nesta perspectiva, a organização do ensino superior deve assentar nos pressupostos das teorias do desenvolvimento do estudante.

As competências cognitivas são, entre muitas outras, pilares fundamentais num processo de valorização da formação do novo diplomado. É neste enquadramento que o presente trabalho pretende saber em que medida se registam diferenças no desenvolvimento cognitivo entre caloiros e finalis- 
tas da Universidade de Coimbra. Elegemos, como referencial teórico, o modelo de desenvolvimento intelectual e ético do estudante do ensino superior, proposto por William Perry (1970), que se insere nas teorias cognitivo-estruturalistas, cuja designação radica na matriz piagetiana e que explicam o desenvolvimento focalizando-se na forma como os indivíduos pensam e atribuem significado às experiências por que vão passando. Apesar de algumas diferenças relativas ao momento das aquisições, a sequência do desenvolvimento é universal, ou seja, independente da cultura, nacionalidade ou meio social de pertença.

Entre muitos outros autores que, segundo Pascarella e Terenzini (2005), se distinguiram no grupo das teorias cognitivo-estruturalistas do desenvolvimento cognitivo do estudante do ensino superior (Lawrence Kohlberg, Carol Gilligan, Marcia Baxter-Magolda, Patricia King e Karen Kitchener), William Perry (1970) foi o pioneiro, com os estudos longitudinais efectuados pela sua equipa, entre as décadas de 50 e 60 do Século XX, com alunos da Universidade de Harvard. Os autores partiram do pressuposto que o nível de desenvolvimento cognitivo determina a forma de encarar as experiências durante o ensino superior e é independente das classificações e das aptidões escolares. Nesta perspectiva, as nove posições desenvolvimentais, que se distribuem por três níveis, não estão sujeitas a limites etários, possibilitam a representação da tendência dominante entre as estruturas e apresentam-se como estáticas. O dinamismo do desenvolvimento é, na perspectiva do autor, conferido pelas transições, e assenta nos quatro conceitos básicos de dualismo, multiplicidade, relativismo e compromisso. Quanto ao dualismo, pretende significar a visão dicotómica do mundo, que opõe o certo ao errado, o bom ao mau e o verdadeiro ao falso, que transforma o aluno num mero aprendiz que memoriza e repete conhecimentos transmitidos por autoridades de competência reconhecida (professores, livros, etc.). Em determinado momento, começa a ser possível admitir diferentes pontos de vista em áreas ou questões para as quais se desconhecem, ainda, as respostas correctas (multiplicidade). No entanto, não há espaço para a formulação de juízos de valor e as opiniões são consideradas de uma forma atomista, sendo igualmente válidas e acertadas. Ao admitir-se o carácter relativo das opiniões e conhecimentos, reconhece-se a necessidade de os fundamentar e submeter a juízos de valor sobre pontos de vista alternativos (relativismo). Os referenciais teóricos deixam de ser considerados como unidades soltas de conhecimento não organizado e passam a conjuntos de ideias com capacidade explicativa e preditora, ao mesmo tempo que os conceitos substituem os factos. Agora o estudo é mais selectivo e rentabilizado com o fundamental, em detrimento do acessório e irrelevante. Finalmente, depois de aceitar o relativismo, o estudante consegue assumir compromissos com posições tomadas (compromisso). Inicia-se aqui o desenvolvimento ético a que se refere Perry, 
que se estende por diferentes áreas de conteúdo (educação, religião, política, carreira, etc.), ainda que sem um desenvolvimento simultâneo do compromisso, que se traduz em desfasamentos horizontais.

No modelo de Perry (1970) o estudante tem um papel activo, num processo em que são possíveis desvios ao desenvolvimento, traduzido pelos conceitos de adiamento (temporizing, adiar o movimento, permanecendo um ano ou mais na mesma posição), fuga (escape, alienação e abandono da responsabilidade, através do refúgio no relativismo e evitamento do compromisso) e retracção (retreat, regresso ao dualismo por evitamento da complexidade e ambivalência). As causas podem ser várias, mas assentam, fundamentalmente, na pressão exagerada exercida pelo contexto: o regresso ao dualismo em consequência de uma aprendizagem mecânica, de forma a atingir o êxito ou a manutenção no relativismo, enquanto forma segura e protectora das escolhas inerentes ao compromisso.

Pascarella e Terenzini (1991, 2005), na sua síntese sobre três décadas de investigação americana, põem em evidência um impacto positivo da frequência do ensino superior, com os finalistas a distinguirem-se comparativamente aos caloiros, no pensamento crítico (ganhos de $.50 D P$ a $1 D P$ ), na disposição para pensar criticamente $(.50 \mathrm{DP})$, na utilização da razão e de provas para encarar problemas não estruturados para os quais não há respostas certas (ganhos de $1 D P$ ), na flexibilidade intelectual e na capacidade para uma abordagem da complexidade conceptual a partir da abstracção (ganhos na ordem de $1.2 \mathrm{DP}$ ), no pensamento formal piagetiano (ganhos de $.33 \mathrm{DP}$ ). No que se refere ao pensamento ou raciocínio pós-formal, a melhor estimativa de magnitude dos efeitos, no julgamento reflexivo é de $.90 \mathrm{DP}$ e na sofisticação epistemológica ou maturidade de $2 \mathrm{DP}$. Os autores partem do pressuposto que uma parte significativa dos ganhos durante o ensino superior, no pensamento crítico e no raciocínio pós-formal, é exclusivamente atribuível à exposição a este nível de ensino, esquecendo os efeitos da maturação que podem estar presentes. Também a selectividade institucional se mostra, nalguns casos, uma variável relacionada com as competências cognitivas gerais e o desenvolvimento intelectual dos alunos, ainda que este efeito seja superior no primeiro ano e com tendência a esbater-se e a desaparecer no final do terceiro. Por outro lado, as investigações apontam no sentido de um impacto selectivo do campo ou área de estudos. Assim, há estudos que mostram uma relação positiva significativa entre a exposição à área de Ciências Naturais e as capacidades de pensamento crítico, apesar de, noutras áreas, os resultados serem inconclusivos ou necessitarem de replicação. Quando tomadas em consideração as características individuais dos alunos, a investigação apresenta efeitos diferenciados do ensino superior em função do género e raça, por exemplo.

Incomparáveis, quantitativamente, com a realidade americana, os trabalhos portugueses sobre o impacto do ensino superior no desenvolvimento 
dos estudantes começam a intensificar-se. Se, em 1995, Ferreira e Bastos se referiam à escassez de estudos neste domínio em Portugal e destacavam seis trabalhos (Barbosa, 1992; Bastos, 1993; Bastos \& Gonçalves, 1994; Ferreira, 1990; Ferreira \& Hood, 1991; Ribeiro, 1993), hoje encontramos outras referências importantes, que podem servir de trampolim para o incremento da pesquisa na nossa realidade. Na sua maioria, é destacável o impacto positivo do ensino superior no desenvolvimento do pensamento pós-formal, com melhorias que se traduzem, fundamentalmente, numa diminuição do dualismo e menores variações no relativismo e no compromisso (Bastos, 1998; Faria \& Bastos, 2005; Ferreira \& Bastos, 1995; Medeiros et al., 2002; Pereira e Medeiros, 2005), bem como o efeito do género (Bastos, 1998; Faria \& Bastos, 2005; Ferreira \& Bastos, 1995) e do curso ou estabelecimento frequentados (Bastos, 1998; Martins, 2005; Medeiros et al., 2002). No entanto, assistimos a alguns casos em que não se registam alterações significativas, ou se encontram mesmo valores de pensamento inferiores nos finalistas (Bastos et al., 2003; Martins, 2005; Pereira \& Medeiros, 2005), o que é explicado pelos autores pela proximidade com o mercado de trabalho e a consequente percepção da redução do leque de possibilidades de mudança (Bastos et al., 2003; Martins, 2005), ou pela organização da formação nos períodos finais no curso de Enfermagem (Pereira \& Medeiros, 2005). Com efeito, o próprio Perry (1970) previu, no seu modelo, a possibilidade de regressões ou paragens em virtude de pressões externas.

\section{Metodologia}

\section{Participantes}

O estudo incluiu 824 participantes, alunos de licenciatura a frequentar o primeiro (505) e último (319) anos de seis cursos da Universidade de Coimbra $^{2}$, no ano lectivo de 2003/2004. A distribuição dos participantes por estabelecimento/curso, género e ano curricular encontra-se no Quadro 1. É uma amostra de conveniência, maioritariamente feminina, correspondendo às características da população abrangida.

\footnotetext{
2 Faculdade de Psicologia e de Ciências da Educação (FacPsicologia), Faculdade de Direito (FacDireito), Faculdade de Economia (FacEconomia), Faculdade de Letras (FacLetras) e Faculdade de Ciências Farmacêuticas (FacFarmácia)
} 
Quadro 1. Distribuição dos participantes por estabelecimento e curso, em função do género (frequências e \%) e ano curricular.

\begin{tabular}{|c|c|c|c|c|c|c|}
\hline \multirow[t]{3}{*}{ Curso } & \multicolumn{4}{|c|}{ Género } & \multirow{2}{*}{\multicolumn{2}{|c|}{ Total }} \\
\hline & \multicolumn{2}{|c|}{$\mathbf{F}$} & \multicolumn{2}{|c|}{$\mathbf{M}$} & & \\
\hline & $\mathrm{N}$ & $\%$ & $\mathrm{~N}$ & $\%$ & $\mathrm{~N}$ & $\%$ \\
\hline \multicolumn{7}{|l|}{ FacPsicologia } \\
\hline Psicologia & $154(110 / 44)$ & 91,1 & $15(12 / 3)$ & 8.9 & $169(122 / 47)$ & 20.5 \\
\hline C. da Educação & $78(50 / 28)$ & 82,9 & $16(10 / 6)$ & 17.1 & $94(60 / 34)$ & 11.4 \\
\hline \multicolumn{7}{|l|}{ FacDireito } \\
\hline Direito & $139(77 / 62)$ & 75.9 & $44(24 / 20)$ & 24.1 & $183(101 / 82)$ & 22.2 \\
\hline \multicolumn{7}{|l|}{ FacEconomia } \\
\hline Economia & $35(26 / 9)$ & 61.4 & $22(17 / 5)$ & 38.6 & $57(43 / 14)$ & 6.9 \\
\hline Gestão & $29(15 / 14)$ & 58 & $21(8 / 13)$ & 42 & $50(23 / 27)$ & 6.1 \\
\hline \multicolumn{7}{|l|}{ FacLetras } \\
\hline História & $66(38 / 28)$ & 63.5 & $38(21 / 17)$ & 36.5 & $104(59 / 45)$ & 12.6 \\
\hline Geografia & $51(28 / 23)$ & 60.7 & $33(29 / 4)$ & 39.3 & $84(57 / 27)$ & 10.2 \\
\hline \multicolumn{7}{|l|}{ FacFarmácia } \\
\hline Farmácia & $42(19 / 23)$ & 76.4 & $13(8 / 5)$ & 23.6 & $55(27 / 28)$ & 6.7 \\
\hline \multicolumn{7}{|l|}{ FacCiências } \\
\hline Biologia & $20(9 / 11)$ & 71.4 & $8(4 / 4)$ & 28.6 & $28(13 / 15)$ & 3.4 \\
\hline Totais & $614(372 / 242)$ & & $210(133 / 77)$ & & $824(505 / 319)$ & \\
\hline
\end{tabular}

Nota: Os valores entre parênteses referem-se às frequências de 'caloiros/finalistas.'

\section{Variáveis}

Dependentes (VD): Pensamento, operacionalizado pelos índices de dualismo, relativismo e compromisso dos conteúdos Carreira e Educação do IDCP, bem como por dois índices finais (um por conteúdo), calculados a partir da diferença entre o somatório dos índices de compromisso e relativismo e o índice de dualismo $($ IDCP Final $=[$ relativismo + compromisso $]-$ dualismo $)^{3}$.

\footnotetext{
${ }^{3}$ Relativamente a cada conteúdo (Carreira e Educação) há a considerar três níveis e um índice final: IDCP carreira dualismo, relativismo, compromisso e final (IDCPcd, IDCPcr e
} 
Independentes (VI): Género e ano curricular (caloiros e finalistas).

\section{Procedimento}

Os dados foram recolhidos de Outubro de 2003 a Janeiro de 2004, em aulas disponibilizadas pelas diferentes instituições e professores, não sendo possível escolher espaços lectivos com maior frequência de alunos (o que, normalmente, acontece nas aulas práticas). Permitiu-se aos alunos, depois de esclarecidos sobre os objectivos do estudo, justificada a importância da colaboração, garantida a confidencialidade e efectuados alguns esclarecimentos, que saíssem da sala se não pretendessem colaborar.

\section{Instrumentos}

Utilizaram-se as subescalas de Educação e Carreira (num total de 50 itens cada) do Inventário de Desenvolvimento Cognitivo de Parker (IDCP), validado e aferido para a população portuguesa por Ferreira e Bastos (1995).

\section{Resultados}

\section{Comparação entre géneros}

Apesar do padrão similar entre géneros, a análise dos resultados (Quadro 2) permite-nos constatar um resultado claramente favorável às raparigas. Assim, o género feminino apresenta valores médios de dualismo (nos conteúdos Carreira e Educação) mais baixos e os de relativismo e compromisso mais elevados. Essas diferenças só não são estatisticamente significativas no compromisso e no relativismo da Educação. No IDCP Final, as diferenças inter género mantêm-se.

\section{Comparação entre caloiros e finalistas}

A comparação entre os índices de pensamento de caloiros e finalistas permite-nos perceber o impacto positivo, esperado, da frequência do ensino superior no desenvolvimento cognitivo (Quadro 3). Com efeito, constatamos diferenças estatisticamente significativas em quase todos os níveis das duas dimensões do IDCP (excepção para o compromisso), com os alunos finalistas a apresentarem um perfil de pensamento mais evoluído (menos dualistas

IDCPcc e IDCPfc) e IDCP educação dualismo, relativismo e final (IDCPed, IDCPer e IDCPec e IDCPfe). 
e mais relativistas). Os valores moderados da magnitude dos efeitos ${ }^{4}$ relativa aos índices finais resultam, fundamentalmente, da evolução no pensamento dualista, uma vez que as subidas no relativismo são fracas em unidades de desvio padrão (não superiores a $.20 \mathrm{DP}$ ). O padrão evolutivo parece, assim, evidenciar que as melhorias começam por se dar nos níveis inferiores e, progressivamente, se estendem às posições superiores do pensamento.

Quadro 2. Comparação de médias entre géneros (magnitude dos efeitos e teste $t$ ) no IDCP.

\begin{tabular}{|c|c|c|c|c|c|c|c|c|c|c|}
\hline & & \multicolumn{3}{|c|}{ MASCULINO } & \multicolumn{3}{|c|}{ FEMININO } & \multirow[b]{2}{*}{ gl } & \multirow[b]{2}{*}{$t$} & \multirow[b]{2}{*}{$p$} \\
\hline \multicolumn{2}{|c|}{ IDCP } & $N$ & $M$ & $D P$ & $N$ & $M$ & $D P$ & & & \\
\hline \multirow[t]{2}{*}{ Dualismo } & Carreira & 201 & 35.62 & 3.76 & 588 & 34.97 & 3.82 & 787 & -2.068 & .039 \\
\hline & Educação & 196 & 40.12 & 5.85 & 591 & 38.75 & 5.31 & 785 & -3.051 & .002 \\
\hline \multirow[t]{2}{*}{ Relativismo } & Carreira & 198 & 53.58 & 4.72 & 585 & 54.51 & 4.56 & 781 & 2.460 & .014 \\
\hline & Educação & 199 & 50.01 & 4.11 & 589 & 50.64 & 4.20 & 786 & 1.847 & .065 \\
\hline \multirow[t]{2}{*}{ Compromisso } & Carreira & 198 & 51.34 & 4.52 & 587 & 52.01 & 4.11 & 783 & 1.906 & .057 \\
\hline & Educação & 197 & 50.17 & 4.32 & 596 & 50.43 & 4,08 & 791 & .773 & .440 \\
\hline \multirow[t]{2}{*}{ Final } & Carreira & 194 & 69.38 & 10.06 & 559 & 71.65 & 9.43 & 751 & 2.830 & .005 \\
\hline & Educação & 190 & 60.19 & 10.14 & 570 & 62.38 & 9.87 & 758 & 2.598 & .010 \\
\hline
\end{tabular}

Estas diferenças são confirmadas pela análise da magnitude dos efeitos, em unidades de desvio padrão, com ganhos moderados no dualismo (-.49 DP e -.51 DP, na Carreira e Educação) e IDCP Final (.35 DP e .34 DP). Nos restantes níveis, os valores são pequenos ou desprezíveis (próximos de zero no compromisso da Educação), ainda que ligeiramente superiores no relativismo. Apesar destes resultados, a natureza do estudo (transversal) não permite concluir, de forma categórica, pelo efeito do ensino superior.

\footnotetext{
${ }^{4}$ A magnitude dos efeitos (effect size) é, habitualmente, estimada em unidades de desvio padrão (DP), a partir do quociente da diferença entre as médias obtidas no primeiro e segundo momentos (ou dos dois grupos) e o desvio padrão global (relativo aos dois momentos ou grupos). Quando o desvio padrão global (pooled) não está disponível, pode utilizar-se o referente ao primeiro momento ou grupo. Apesar de algum desacordo quanto ao significado a conferir aos resultados de obtidos da magnitude dos efeitos, a literatura parece evidenciar maior consenso em considerar pequenos valores inferiores a .30, moderados entre .30 e .70 e elevados acima de .70 (Pascarella \& Terenzini, 2005).
} 
Quadro 3. Comparação de médias caloiros-finalistas (magnitude dos efeitos - ME e teste $t$ ) no IDCP.

\begin{tabular}{|c|c|c|c|c|c|c|c|c|c|c|c|}
\hline & & \multicolumn{3}{|c|}{ CALOIROS } & \multicolumn{3}{|c|}{ FINALISTAS } & \multicolumn{4}{|c|}{ Teste $t$} \\
\hline \multicolumn{2}{|c|}{ Variável dependente (VD) } & $N$ & $M$ & $D P$ & $N$ & $M$ & $D P$ & $M E$ & gl & $t$ & $p$ \\
\hline \multicolumn{12}{|l|}{$\overline{\text { IDCP }}$} \\
\hline \multirow[t]{2}{*}{ Dualismo } & Carreira & 449 & 35.75 & 3.76 & 277 & 33.89 & 3.54 & -.49 & 724 & 6.584 & .000 \\
\hline & Educação & 446 & 39.93 & 5.29 & 279 & 37.24 & 5.33 & -.51 & 722 & -2.692 & .007 \\
\hline \multirow[t]{2}{*}{ Relativismo } & Carreira & 447 & 53.98 & 4.78 & 277 & 54.94 & 4.43 & .20 & 721 & -2.010 & .045 \\
\hline & Educação & 445 & 50.30 & 4.31 & 278 & 50.92 & 4.19 & .14 & 723 & 6.659 & .000 \\
\hline \multirow[t]{2}{*}{ Compromisso } & Carreira & 444 & 51.60 & 4.20 & 279 & 52.25 & 4.19 & .15 & 721 & -1.926 & .054 \\
\hline & Educação & 450 & 50.37 & 4.29 & 279 & 50.49 & 4,00 & .03 & 727 & -.387 & .699 \\
\hline \multirow[t]{2}{*}{ Final } & Carreira & 431 & 69.90 & 9.71 & 269 & 73.31 & 9.23 & .35 & 698 & -4.597 & .000 \\
\hline & Educação & 432 & 60.87 & 9.82 & 271 & 64.24 & 10.07 & .34 & 701 & -4.375 & .000 \\
\hline
\end{tabular}

Uma análise mais fina, que perspectiva as diferenças entre caloiros e finalistas em cada estabelecimento de ensino, evidencia especificidades favoráveis às faculdades de Direito e Psicologia, com alterações positivas no dualismo e, respectivamente, no relativismo e compromisso (Quadro 4). Economia e Letras aparecem a seguir, ainda que os ganhos se traduzam, apenas, no dualismo. Realçamos, também, a faculdade de Letras como o estabelecimento de ensino superior com níveis de pensamento menos evoluídos (mais dualismo e menor relativismo e compromisso). No extremo oposto, encontra-se a faculdade de Farmácia, que não apresenta evolução significativa no pensamento, acontecendo mesmo situações de melhores resultados nos caloiros. Salientam-se os valores elevados de magnitude dos efeitos, por contraste com valores pequenos (inferiores a .30) nos restantes casos. 
Quadro 4. Médias e Desvios-Padrão no IDCP, por estabelecimento de ensino

\begin{tabular}{|c|c|c|c|c|c|c|c|c|c|c|}
\hline ESTAB. & $N$ & $M$ & $D P$ & $M E$ & & ESTAB. & $N$ & $M$ & $D P$ & $M E$ \\
\hline FacPsicologia* & 229 & 33.95 & 3.57 & -.30 & \multirow{6}{*}{$\begin{array}{l}\text { Dे } \\
\text { Оे } \\
\text { Ө }\end{array}$} & FacPsicologia* & 225 & 36.84 & 5.15 & -.48 \\
\hline FacDireito* & 154 & 35.78 & 3.80 & -.73 & & FacDireito* & 157 & 39.89 & 5.79 & -.62 \\
\hline FacEconomia* & 98 & 35.21 & 3.96 & -.84 & & FacEconomia & 98 & 39.83 & 5.06 & -.51 \\
\hline FacLetras* & 168 & 36.06 & 3.66 & -.5 & & FacLetras* & 169 & 40.09 & 5.53 & -.81 \\
\hline Ë FacFarmácia & 50 & 34.52 & 3.87 & .22 & & FacFarmácia & 51 & 38.84 & 4.39 & .29 \\
\hline FacCiências & 27 & 34.15 & 2.88 & -.48 & & FacCiências & 25 & 39.60 & 4.18 & -.40 \\
\hline FacPsicologia & 227 & 54.95 & 5.16 & .20 & \multirow{6}{*}{$\begin{array}{l}\dot{0} \\
\stackrel{0}{\Theta}\end{array}$} & FacPsicologia & 228 & 50.95 & 4.42 & -.03 \\
\hline FacDireito* & 152 & 54.71 & 4.13 & .14 & & FacDireito & 155 & 50.98 & 4.12 & -.04 \\
\hline FacEconomia & 97 & 54.26 & 4.63 & -.08 & & FacEconomia & 97 & 49.99 & 4.37 & .07 \\
\hline FacLetras & 169 & 52.95 & 4.31 & .11 & & FacLetras & 167 & 49.77 & 4.04 & .09 \\
\hline FacFarmácia & 52 & 55.17 & 4.32 & .07 & & FacFarmácia & 48 & 50.94 & 3.70 & .12 \\
\hline FacCiências & 27 & 54.81 & 4.85 & .24 & & FacCiências & 28 & 50.75 & 3.96 & .06 \\
\hline FacPsicologia* & 227 & 52.27 & 4.31 & .31 & \multirow{6}{*}{ 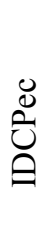 } & FacPsicologia & 227 & 50.38 & 4.34 & -.05 \\
\hline FacDireito & 153 & 52.31 & 3.93 & -.28 & & FacDireito & 156 & 50.76 & 4.19 & -.09 \\
\hline FacEconomia & 96 & 51.01 & 4.13 & -.06 & & FacEconomia & 98 & 50.19 & 3.91 & -.02 \\
\hline FacLetras & 169 & 51.43 & 4.05 & .09 & & FacLetras & 171 & 50.12 & 4.17 & -.28 \\
\hline FacFarmácia & 51 & 51.49 & 4.22 & .01 & & FacFarmácia & 49 & 50.86 & 3.22 & -.07 \\
\hline FacCiências & 27 & 52.11 & 5.54 & .23 & & FacCiências & 28 & 50.68 & 5.29 & .02 \\
\hline
\end{tabular}

Nota: * Estabelecimentos em que se registaram diferenças significativas entre caloiros e finalistas no teste $t$

As diferenças significativas inter-género e a distribuição desigual dos alunos e alunas por curso levaram-nos a controlar aquela variável (Quadro 5), o que permitiu observar algumas alterações relativamente aos resultados por ano curricular. 
Quadro 5. Comparação (teste $t$ ), por ano curricular: Caloiros (C) e Finalistas (F), das médias no IDCP, controlando o género.

\begin{tabular}{|c|c|c|c|c|c|c|c|c|}
\hline VD & Género & & $N$ & $M$ & $D P$ & $g I$ & $t$ & $p$ \\
\hline \multirow[t]{4}{*}{ IDCPcd } & Fem. & $\mathrm{C}$ & 358 & 35.68 & 3.88 & \multirow{2}{*}{586} & \multirow{2}{*}{5.744} & \multirow{2}{*}{.000} \\
\hline & & $\mathrm{F}$ & 230 & 33.87 & 3.46 & & & \\
\hline & \multirow{2}{*}{ Masc. } & $\mathrm{C}$ & 128 & 36.34 & 3.78 & \multirow{2}{*}{199} & \multirow{2}{*}{3.642} & \multirow{2}{*}{.000} \\
\hline & & $\mathrm{F}$ & 73 & 34.36 & 3.64 & & & \\
\hline \multirow[t]{4}{*}{ IDCPcr } & Fem. & C & 355 & 54.34 & 4.69 & \multirow{2}{*}{583} & \multirow{2}{*}{-1.166} & \multirow{2}{*}{.244} \\
\hline & & $\mathrm{F}$ & 230 & 54.79 & 4.36 & & & \\
\hline & \multirow{2}{*}{ Masc. } & $\mathrm{C}$ & 126 & 52.89 & 4.56 & \multirow{2}{*}{196} & \multirow{2}{*}{-2.758} & \multirow{2}{*}{.006} \\
\hline & & $\mathrm{F}$ & 72 & 54.79 & 4.80 & & & \\
\hline \multirow[t]{4}{*}{ IDCPcc } & Fem. & $\mathrm{C}$ & 355 & 51.82 & 4.16 & \multirow{2}{*}{585} & \multirow{2}{*}{-1.325} & \multirow{2}{*}{.186} \\
\hline & & $\mathrm{F}$ & 232 & 52.28 & 4.04 & & & \\
\hline & \multirow{2}{*}{ Masc. } & C & 126 & 51.16 & 4.32 & \multirow{2}{*}{196} & \multirow{2}{*}{-.747} & \multirow{2}{*}{.456} \\
\hline & & $\mathrm{F}$ & 72 & 51.66 & 4.87 & & & \\
\hline \multirow[t]{4}{*}{ IDCPed } & Fem. & C & 358 & 39.81 & 5.11 & \multirow{2}{*}{589} & \multirow{2}{*}{6.225} & \multirow{2}{*}{.000} \\
\hline & & $\mathrm{F}$ & 233 & 37.11 & 5.20 & & & \\
\hline & Masc. & C & 125 & 40.96 & 5.59 & 194 & 2728 & 007 \\
\hline & & $\mathrm{F}$ & 71 & 38.63 & 5.40 & & & \\
\hline IDCPer & Fem. & C & 357 & 50.47 & 4.31 & 587 & -1.198 & 231 \\
\hline & & $\mathrm{F}$ & 232 & 50.90 & 4.01 & 501 & & \\
\hline & Masc. & $\mathrm{C}$ & 127 & 49.75 & 4.04 & 197 & -1158 & 248 \\
\hline & & $\mathrm{F}$ & 72 & 50.45 & 4.22 & & & \\
\hline IDCPec & Fem. & C & 362 & 50.46 & 4.20 & 594 & 248 & 804 \\
\hline & & $\mathrm{F}$ & 234 & 50.38 & 3.90 & & & \\
\hline & Masc. & C & 126 & 50.00 & 4.32 & 195 & -710 & 478 \\
\hline & & $\mathrm{F}$ & 71 & 50.46 & 4.33 & 193 & -.710 & \\
\hline IDCPfc & Fem. & C & 337 & 70.64 & 9.61 & & -3114 & 007 \\
\hline & & $\mathrm{F}$ & 222 & 73.17 & 8.97 & דכ & -3.114 & .002 \\
\hline & Masc. & C & 124 & 67.75 & 9.49 & 192 & -3064 & 002 \\
\hline & & $\mathrm{F}$ & 70 & 72.27 & 10.45 & 192 & -3.004 & .002 \\
\hline IDCPfe & Fem. & C & 343 & 61.16 & 9.74 & 568 & -3.678 & .000 \\
\hline & & $\mathrm{F}$ & 227 & 64.23 & 9.81 & & & \\
\hline & Masc. & C & 123 & 59.06 & 9.53 & 188 & -2.099 & 037 \\
\hline & & $\mathrm{F}$ & 67 & 62.26 & 10.95 & 100 & -2.099 & ונסט. \\
\hline
\end{tabular}

As diferenças significativas e a tendência das mesmas mantêm-se no comportamento dualista e no IDCP Final, de ambas as subescalas (Carreira e Educação). Porém, no relativismo, as diferenças por ano curricular encontradas na Carreira parecem dever-se, sobretudo, ao género masculino, uma vez que não se registam nas raparigas. Saliente-se, no entanto, que o género 
feminino atinge valores iguais ao masculino, no último ano (54.79), mas parte de níveis mais elevados, no primeiro ano curricular (54.34 contra 52.89).

\section{Discussão dos resultados}

Os resultados, em termos de padrão global de comportamento, são semelhantes aos obtidos na aferição e validação da escala para a população portuguesa (Ferreira \& Bastos, 1995), ou seja, os alunos apresentam valores de pensamento relativista e de compromisso superiores aos de dualismo. No entanto, aquela amostra obteve resultados, genericamente, mais favoráveis, sobretudo na Carreira, com valores de dualismo inferiores e níveis de relativismo e compromisso superiores. No que respeita às diferenças entre géneros, apesar de também beneficiarem as raparigas, elas são mais evidentes e expressivas nos nossos resultados, uma vez que os autores só as encontraram na Carreira (relativismo e compromisso). Outros trabalhos realizados em Portugal, que utilizaram o IDCP, colocaram em evidência a superioridade feminina no pensamento (Bastos, 1998; Martins, 2005), mas Medeiros et al. (2002) concluíram pela inexistência de efeito significativo da variável género. Pires (2001) registou, mesmo, diferenças inter-género na posição relativista da Educação favoráveis aos rapazes. King e Kitchener (1994), que chegaram a resultados inconsistentes sobre o efeito do género no julgamento reflexivo, relatam, numa síntese de quarenta estudos, apenas sete com diferenças significativas, favoráveis aos homens em seis casos.

Os resultados a que chegámos enquadram-se num dos pressupostos teóricos que tem vindo a ser corroborado pela investigação e que defende a evolução do pensamento ao longo do ensino superior, através da diminuição do dualismo e do aumento do relativismo e do compromisso (Bastos, 1998; Ferreira \& Bastos, 1995; King \& Kitchener, 1994). No entanto, Ferreira e Bastos (1995) chegaram a diferenças mais evidentes (com significado estatístico em todos os níveis) no conteúdo Carreira do que na Educação (onde apenas existem diferenças significativas no dualismo). De acordo com Sprinthall e Collins (1994), a complexidade dos objectivos dos patamares mais evoluídos (relativismo e compromisso) pode favorecer um aumento do período temporal em que se desenvolvem. Igualmente, para King e Kitchener (1994), espera-se que as modificações comecem por acontecer no nível mais elementar (dualista) e progressivamente se estendam aos restantes, uma vez que o desenvolvimento do julgamento reflexivo se processa de forma sequencial (cada estádio edifica-se no anterior e alicerça-se no seguinte). Também Baxter-Magolda (1992) conclui pela diminuição progressiva do pensamento absoluto durante o ensino superior, à medida que aumentam os 
restantes (sobretudo de transição e independente), enquanto o conhecimento contextualizado sobe nos últimos anos da licenciatura e nas pós-graduações. No mesmo sentido, Faria e Bastos (2005) identificaram o modo de conhecimento absoluto, nos dois primeiros anos e de transição, no terceiro ano.

Outros trabalhos realizados em Portugal concluíram de modo um pouco diferente. Assim, Medeiros et al. (2002) registaram posições menos dualistas ao longo dos quatro anos de frequência do ensino superior, mas verificaram, também, estabilidade no relativismo e compromisso, ou mesmo oscilações onde se incluem descidas (regressões). Igualmente, Bastos et al. (2005) confrontaram-se, ao longo de um ano, com descidas nas únicas diferenças significativas que obtiveram (subescala Carreira). Porém, o facto de se tratar de uma amostra de finalistas, pode remeter para os efeitos descritos por Perry (1970), aos quais já aludimos.

Estudos americanos, revistos por Pascarella e Terenzini (1991, 2005), põem em evidência níveis de desenvolvimento superiores, à medida que se avança na escolaridade, quer seja no pensamento crítico (Beck et al., 1992; Brooks \& Shepard, 1990; Dressel \& Mayhew, 1954; Drouin, 1992; Hill, 1995; Keely, Brown \& Kreutzer, 1982; Lehmann, 1963, 1968; McDonough, 1997; McMillan, 1987; Mines et al., 1990; Pascarella, 1999; Pearson, 1991; Steele, 1986; Watson \& Glaser, 1980), na complexidade conceptual e na aprendizagem de novos conceitos (Flowers et al., 2001; Khalili \& Hood, 1983; Meyer, 1977), no pensamento pós-formal, segundo o esquema de Perry (Baxter-Magolda, 1988; Baxter-Magolda \& Porterfield, 1985; Blake, 1976; Ferreira \& Bastos, 1995; Martins, 2005; Mentkowski \& Strait, 1983; Meyer, 1977; Moore, 1989; Whitla, 1978; Winter et al., 1981), no julgamento reflexivo (Baxter-Magolda, 1988; King \& Kitchener, 1994; Kitchener et al., 1989; Kitchener \& Wood, 1987; Mines et al., 1990; Wood, 2000) e no raciocínio informal (Perkins, 1985; Perkins et al., 1986).

Não obstante a vasta concordância nesta matéria, há autores que obtêm resultados divergentes, sobretudo com a introdução de variáveis de controlo. É o caso de Parker e Thorndike (1989) que verificaram uma minimização das diferenças entre vários níveis curriculares no ensino superior, quando controlaram a idade e de Jensen et al. (1999), que viram desaparecer as diferenças no pensamento crítico com o controlo da aptidão verbal. Também a raça e o género se evidenciaram como variáveis mediadoras, quer em estudos de Terenzini et al. (1994) e de Flowers (2000), com os maiores ganhos de pensamento crítico, respectivamente, em alunos euro-americanos não latinos e euro-americanos, quer através do impacto significativo do estatuto sócio-económico, apenas no género feminino, num trabalho de Whitt et al. (2003).

Igualmente, as características institucionais, sobretudo pela diversidade de experiências e vivências proporcionadas, têm-se revelado como dife- 
renciadoras do impacto do ensino superior no desenvolvimento cognitivo (Pace, 1997; Pascarella \& Terenzini, 2005; Smart, 1997; Smart \& Feldman, 1998). Também nos estabelecimentos considerados no nosso estudo se assiste a uma evolução distinta do pensamento dos seus alunos.

Em termos de análises da magnitude dos efeitos, genericamente, a literatura apresenta ganhos no desenvolvimento cognitivo, durante o ensino superior, mais elevados que os do presente estudo, ainda que, em alguns casos os resultados se aproximem (Hagedorn et al., 1999 apresentam valores que variam entre $30 \mathrm{DP}$ e $.40 \mathrm{DP})$. Da primeira para a segunda síntese de Pascarella e Terenzini $(1991,2005)$ ressalta uma tendência para menores incrementos, o que pode dever-se à antecipação do desenvolvimento, por alteração das condições educativas e culturais inerentes às experiências vividas pelos alunos ao longo da vida, levando a melhores níveis à entrada para o ensino superior e, consequentemente, a progressos mais lentos e difíceis. Os ganhos no pensamento crítico variam entre $.25 D P$ (Mentkowski et al., 1991) e $1 D P$ (Lehmann, 1963; 1968), apesar dos ganhos inferiores (.15 DP) obtidos por Facione (1997), com alunos de Enfermagem, entre o segundo e o último ano.

Os ganhos no pensamento pós-formal variam entre .40 DP (Mentkowski \& Strait, 1983) e $1.9 D P^{5}$ (King \& Kitchener, 1994), em estudos de natureza transversal e longitudinal, respectivamente.

\section{Conclusão}

Não só pela acessibilidade, mas também pela riqueza do contexto em que se move, a população de estudantes do ensino superior tem sido alvo de grande interesse da investigação, sobretudo norte-americana, que se preocupa com o desenvolvimento do jovem adulto/adulto emergente. Com efeito, o estudo do impacto do ensino superior no desenvolvimento dos alunos tem permitido confundir, por vezes, uma etapa do desenvolvimento humano (jovem adulto) com o desenvolvimento em contexto daqueles que têm oportunidade de lhe aceder. Em nossa opinião, não deve ser este o ponto fulcral de discussão, antes retirar daqueles trabalhos os contributos importantes para fazer do ensino superior um contexto realmente potenciador do desenvolvimento global dos seus alunos. Os desafios que actualmente se colocam a este sistema de ensino aumentam a pertinência deste desiderato.

É neste enquadramento que surge o estudo aqui apresentado e que incide sobre o impacto da frequência do ensino superior no pensamento, em

\footnotetext{
${ }^{5}$ Valor calculado por Pascarella e Terenzini (2005).
} 
alunos da Universidade de Coimbra. Os resultados, ressalvadas as limitações decorrentes da natureza transversal do estudo e das características da amostra disponível, evidenciam um efeito positivo do ensino superior no desenvolvimento cognitivo, sobretudo porque os alunos se tornam menos dualistas. Os ganhos inferiores no relativismo podem traduzir a forma sequencial como Perry (1970) definiu o desenvolvimento. No que respeita à ausência de diferenças significativas no compromisso, alguns autores defendem que não é claro falar-se do compromisso como um nível de desenvolvimento cognitivo exclusivamente, pois pode estar implicada uma dimensão associada ao desenvolvimento da identidade (King \& Kitchener, 1994; Ferreira, 2000).

Porém, parece importante uma reflexão sobre a possibilidade de o ensino superior se constituir como um contributo mais decisivo, no relativismo e compromisso, verificando quais as formas de organização do ensino mais favoráveis. Aqui, assumem grande relevo os currículos, métodos de ensino e avaliação, modelos de organização, possibilidades de participação e de envolvimento dos alunos, etc.. Pereira e Medeiros (2005) defendem, por exemplo, que a formação deve privilegiar um perfil de profissional que requer, frequentemente, tomadas de decisão independentes e críticas, numa dimensão auto-formativa e não de execução de tarefas, ainda que técnicas.

É fundamental replicar o estudo aqui apresentado, como também recorrer a trabalhos que acompanhem os alunos longitudinalmente e contar com o contributo de metodologias qualitativas que permitam compreender as especificidades de cada contexto e favoreçam intervenções diferenciadas.

\section{Referências}

Almeida, L., \& Santos, L. (1999). Adaptação e rendimento académico: Estudo com alunos universitários do $1^{\circ}$ ano. In A. Soares, S. Araújo \& S. Caires (Orgs.), Avaliação psicológica: Formas e contextos (Vol. 6, pp. 73-80). Braga: APPORT.

Arnett, J. J. (2000). A theory of development from the late teens through the twenties. American Psychologist, 55, 469-480.

Arnett, J. J. (2002). Developmental sources of crash risk in young drivers. Injury Prevention, 8, 17-23.

Arnett, J. J. (2004). Emerging adulthood: The winding road from the late teens through the twenties. Oxford: University Press.

Bastos, A. (1998). Desenvolvimento pessoal e mudança em estudantes do ensino superior: Contributos da teoria, investigação e intervenção. Tese de doutoramento não publicada. Universidade do Minho, Braga.

Bastos, A., Faria, C., Silva, C., Carvalho, I., \& Gonçalves, T. (2003). Desenvolvimento cognitivo e dilemas da prática educativa: Contribuições da investi- 
gação para a qualidade de ensino. Fases, 2, Artigo 5. Consultado em Março 15, 2006, de http://www.ualg.pt/fchs/ceduc/fases/menu2.htm

Bastos, A. Faria, C., Soares, I., Ferreira, J., Medeiros, T., \& Silva, C. (2005). Auto-estima, vinculação e desenvolvimento cognitivo em estudantes do ensino superior português: Um estudo exploratório. In A. Bastos (Coord.), Na arena dos (des) emparelhamentos construtivos: Contribuições da investigação e da intervenção para o desenvolvimento cognitivo e relacional no ensino superior. Relatório de progresso. Programa de apoio a projectos de pesquisa no domínio educativo (2003-2006). Lisboa: Fundação Calouste Gulbenkian.

Baxter-Magolda, M. (1988). The impact of the freshman year on epistemological development: Gender differences. Paper presented at the Meeting of the American Educational Research Association, New Orleans.

Baxter-Magolda, M. (1992). Knowing and reasoning in college: Gender related patterns in students' intellectual development. San Francisco: Jossey-Bass.

Baxter-Magolda, M., \& Porterfield, W. (1985). A new approach to assess intellectual development on the Perry scheme. Journal of College Student Personnel, 26, 343-350.

Beck, S., Bennett, A., McLeod, R., \& Molyneaux, D. (1992). Review of research on critical thinking in nursing education. Review of Research in Nursing Education, 5, 1-30.

Belenky, M., Clinchy, B., Goldberg, N., \& Tarule, J. (1986). Women's ways of knowing: The development of self, voice and mind. New York: Basic Books.

Blacke, L. (1976). A major of developmental change: A cross-sectional study. Paper presented at the Annual Meeting of the American Psychological Association. Washington, DC.

Brooks, K., \& Shepard, J. (1990). The relationship between clinical decision-making skills in nursing and general critical thinking abilities of senior nursing students in four types of nursing programs. Journal of Nursing Education, 2, 391-399.

Chikering, A. (1969). Education and identity. San Francisco: Jossey-Bass.

Chickering, A., \& Reisser, L. (1993). Education and identity. San Francisco: Jossey-Bass Publishers.

Dressel, P., \& Mayhew, L. (1954). General education: Explorations in evaluation. Westport, CT: Greenwood Press.

Drouin, L. (1992). An investigation of the critical thinking ability of engineering students seeking a Bachelor of Science degree. Dissertation Abstracts International, 53, 1070A.

Erikson, E. (1959). Identity and the life cycle. New York: Norton.

Erikson, E. (1968). Identity: Youth and crisis. New York: Norton.

Facione, N. (1997). Critical thinking assessment in nursing education programs: An aggregate data analysis. Millbrae, CA: California Academic Press.

Faria, C., \& Bastos, A. (2005). Desenvolvimento epistemológico em estudantes do ensino superior português. In A. Bastos (Coord.), Na arena dos (des)emparelhamentos construtivos: Contribuições da investigação e da intervenção para o desenvolvimento cognitivo e relacional no ensino superior. Relatório de pro- 
gresso. Programa de apoio a projectos de pesquisa no domínio educativo (2003-2006). Lisboa: Fundação Calouste Gulbenkian.

Ferreira, J. (2000). O desenvolvimento psicológico do jovem adulto em contexto de ensino universitário. Lição de síntese apresentada à Faculdade de Psicologia e de Ciências da Educação, Universidade de Coimbra.

Ferreira, J., \& Bastos, A. (1995). Inventário de Desenvolvimento Cognitivo de Parker. In L. Almeida, M. Simões \& M. Gonçalves (Eds.), Provas psicológicas em Portugal (Vol. 1, pp. 287-307). Braga: APPORT.

Flowers, L. (2000). Cognitive effects of college: Differences between African-American and Caucasian students. Unpublished doctoral dissertation, University of Iowa, Iowa City.

Flowers, L., Osterlind, S., Pascarella, E., \& Pierson, C. (2001). How much do students learn in college? Cross-sectional estimates using the college BASE. The Journal of Higher Education, 72(5), 565-583. (ProQuest Document Reproduction Service $\mathrm{N}^{\mathrm{o}}$ 00221546).

Gould, R. (1978). Transformations: Growth and change in adult life. New York: Simon \& Schuster.

Hagedorn, L., Pascarella, E., Edison, M., Braxton, J., Nora, A., \& Terenzini, P. (1999). Institutional context and the development of critical thinking: A research note. Review of Higher Education, 22, 247-263.

Havigurst, R. (1973). Developmental tasks and education ( $3^{\text {rd }}$ ed.). New York: David McKay Company, Inc.

Heath, D. (1965). Explorations of maturity: Studies of mature and immature college men. New York: Appleton-Century-Crofts.

Hill, K. (1995). Critical thinking and its relation to academic, personal, and moral development in the college years. Dissertation Abstracts International, 56, 4603B.

Hood, A., \& Ferreira, J. (1983). Stages in the cognitive development of university students. Separata da Revista Portuguesa de Pedagogia, XVII, 79-90.

Jensen, L., Kitchener, K., \& Wood, P. (1999). The role of need for cognition in the development of reflective judgment. Paper presented at the Meeting of the American Psychological Association, Boston.

Josselson, R. (1987). Identity diffusion: A long-term follow-up. Adolescent Psychology, 14, 230-258.

Keeley, S., Browne, M., \& Kreutzer, J. (1982). A comparison of freshmen and seniors on general and specific essay tests of critical thinking. Research in Higher Education, 17(2), 139-154.

Keniston, K. (1965). The uncommitted: Alienated youth in American society. New York: Harcourt, Brace \& World.

Keniston, K. (1971). Youth and dissent. New York: Harcourt, Brace \& Jovanovich.

Khalili, H., \& Hood, A. (1983). A longitudinal study of change in conceptual level in college. Journal of College Student Personnel, 24, 389-394.

King, P., \& Kitchener, K. (1994). Developing reflective judgment. San Francisco: Jossey-Bass. 
Kitchener, K., \& King, P. (1981). Reflective judgment: Concepts of justification and their relation to age and education. Journal of Applied Developmental Psychology, 2, 89-116.

Kitchener, K., King, P., Wood, P., \& Davison, M. (1989). Sequentiality and consistency in the development of reflexive judgment: A six year longitudinal study. Journal of Applied Developmental Psychology, 10, 73-95.

Kitchener, K., \& Wood, P. (1987). Development of concepts of justification in Germany university students. International Journal of Behavioral Development, 10, 171-185.

Knefelkamp, L., Widick, C., \& Parker, C. (1978). Editor's notes: Why bother with theory?. In L. Knefelkamp, C. Widick \& C. Parker (Eds.), Applying new developmental findings: New directions for student services, $n^{\circ} 4$ (pp. 7-16). San Francisco: Jossey-Bass.

Köhlberg, L. (1971). Stages of moral development. In C. Beck, B. Crittenden \& E. Sullivan (Eds.), Moral education. Toronto: University of Toronto Press.

Köhlberg, L. (1979). Measuring moral judgment. Worcester, Mass: Clark University Press.

Lehmann, I. (1963). Changes in critical thinking, attitudes, and values from freshman to senior years. Journal of Education Psychology, 54, 305-315.

Lehmann, I. (1968). Changes from freshman to senior years. In K. Yamamoto (Ed.), The college student and his culture. Boston: Houghton Mifflin.

Levinson, D. (1978). The seasons of a man's life. New York: Knopt.

Loevinger, J. (1976). Ego development: Conceptions and theories. San Francisco: Jossey-Bass.

Márcia, J. (1966). Development and validation of ego-identity status. Journal of Personality and Social Psychology, 3, 551-558.

Martins, E. (2005, Abril). O pensamento dos alunos no ensino superior politécnico: Um estudo diferencial em função do género, idade e curso. Comunicação apresentada no VII Congresso da Sociedade Portuguesa de Ciências da Educação: Cenários da educação/formação: Novos espaços, culturas e saberes, Castelo Branco.

Martins, E. (2007). Cognição e desempenho no ensino superior. Tese de doutoramento não publicada, Faculdade de Psicologia e de Ciências da Educação, Universidade de Coimbra.

McDonough, M. (1997). An assessment of critical thinking at the community college level. Dissertation Abstracts International, 58, 2561A.

McMillan, J. (1987). Enhancing college students' critical thinking: A review of studies. Research in Higher Education, 26, 3-29.

Medeiros, T., Ferreira, J., Almeida, L., Peixoto, E., Tavares, J., \& Morais, H. (2002). Desenvolvimento cognitivo do estudante do ensino superior: Efeito do curso, ano e género. Revista Portuguesa de Pedagogia, 36 (1.2.3), 355-373.

Mentkowski, M., Rodgers, G., Deemer, D., Tamar, B., Reisetter, J., Rickards, W., et al. (1991). Understanding abilities, learning, and development through college outcome studies: What can we expect from higher education assessment? 
Paper presented at the Annual Meeting of the American Educational Research Association, Chicago.

Mentkowski, M., \& Strait, M. (1983). A longitudinal study of student change in cognitive development, learning styles, and generic abilities in an outcome-centered liberal arts curriculum (Final report to the National Institute of Education; Research Report No. 6). Milwaukee, WI: Alverno College, Office of Research and Evaluation.

Meyer, P. (1977). Intellectual development: Analysis of religious content. Counseling Psychologist, 6, 47-50.

Mines, R., King, P., Hood, A., \& Wood, P. (1990). Stages of intellectual development and associated critical thinking skills in college students. Journal of College Student Development, 31, 538-547.

Moore, W. (1989). The learning environment preferences: Exploring the construct validity of an objective major of the Perry scheme of intellectual development. Journal of College Student Development, 30, 504-519.

Pace (1997, November). Connecting institutional types to student outcomes. Paper presented at the Annual Meeting of the Association for the Study of Higher Education, Albuquerque, NM.

Parker, J., \& Thorndike, R. (1989). Effects of age and education on cognitive development. Paper presented at the Annual Meeting of the American Educational Research Association, San Francisco.

Pascarella, E. (1999). The development of critical thinking: Does college make a difference?. Journal of College Student Development, 40, 562-569. (ProQuest Document Reproduction Service No 08975264).

Pascarela, E., \& Terenzini, P. (1991). How college affects students: Findings and insights from twenty years of research. San Francisco: Jossey-Bass.

Pascarela, E., \& Terenzini, P. (2005). How college affects students: A third decade of research. San Francisco: Jossey-Bass.

Pearson, C. (1991). Barriers to success: Community college students' critical thinking skills. Washington, DC: U.S. Office of Education. (ERIC Document Reproduction Services No. ED 340415$)$.

Pereira, H., \& Medeiros, M. (2005). Concepções de enfermagem e desenvolvimento cognitivo. In T. Medeiros, \& E. Peixoto (Orgs.), Desenvolvimento e aprendizagem: Do ensino secundário ao ensino superior (pp. 145-156). Açores: Universidade dos Açores.

Perkins, D. (1985). Postprimary education has little impact on informal reasoning. Journal of Educational Psychology, 77, 562-571.

Perkins, D., Bushey, B., \& Farady, M. (1986). Learning to reason (Final Report to the National Institute of Education, Grant NIE-G-83-0028). Cambridge, MA: Harvard University.

Perry, W. (1970). Forms of intellectual and ethical development in the college years. New York: Holt, Rinheart and Winston.

Perry, W. (1981). Cognitive and ethical growth: The making of meaning. In A. Chickering \& Associates, The modern American college: Responding to the 
new realities of diverse students and a changing society. San Francisco: Jossey-Bass.

Pinheiro, M. (2003). Uma época especial: Suporte social e vivências académicas na transição e adaptação ao ensino superior. Tese de doutoramento não publicada, Faculdade de Psicologia e de Ciências da Educação, Universidade de Coimbra.

Pires, H. (2001). Desenvolvimento e adaptação académica em estudantes universitários dos "PALOP". Tese de doutoramento não publicada, Universidade de Évora, Évora.

Rovira, E. (2001). Competencias genéricas en la formación universitaria. Revista de Educação, 325, 229-321.

Sanford, N. (1962). The American college. New York: Atherton Press.

Sanford, N. (1966). Self and society. New York: Atherton Press.

Sanford, N. (1967). Where colleges fail: A study of the student as a person. San Francisco: Jossey-Bass.

Smart, J. (1997). Academic subenvironments and differential patterns of self-perceived growth during college: A test of Holland's theory. Journal of College Student Development, 38, 68-77.

Smart, J., \& Feldman, K. (1998). "Accentuation effects" of dissimilar departments: An application and explanation of Holland's theory. Research in Higher Education, 39, 385-418.

Sprinthall, N., Collins, W. (1994). Psicologia do adolescente: Uma abordagem desenvolvimentista (C. Vieira, Trad.). Lisboa: Gulbenkian.

Steele, J. (1986). Assessing reasoning and communication skills of postsecondary students. Paper presented at the Meeting of the American Educational Research Association, San Francisco.

Terenzini, P., Rendon, L., Upcraft, M., Miller, S., Allison, K., Gregg, P., \& Jalomo, R. (1994). The transition to college: Diverse students, diverse stories. Research in Higher Education, 35, 57-73.

Watson, G., \& Glaser, E. (1980). Watson-Glaser critical thinking appraisal. San Antonio, TX: Psychological Corporation.

Whitla, D. (1978). Value added: Measuring the impact of undergraduate education. Cambridge, MA: Harvard University, Office of Instructional Research and Evaluation.

Whitt, E., Pascarella, E., Neisheim, B., Marth, B., \& Pierson, C. (2003). Differences between women and men in objectively measured outcomes, and the factors that influence those outcomes in the first three years of college. Journal of College Student Development, 44, 587-610.

Winter, D., McClelland, D., \& Stewart, A. (1981). A new case for the liberal arts: Assessing institutions goals and student development. San Francisco: Jossey-Bass.

Wood, P. (2000, August). Scaling and scoring of an objective measure of epistemic development. Paper presented at the Annual Meeting of the American Psychological Association, Washington, DC. 